\title{
On the Leading Energy Correction for the Statistical Model of the Atom: Interacting Case
}

\author{
Heinz Siedentop and Rudi Weikard * \\ Institut für Mathematische Physik, Carolo-Wilhelmina, Mendelssohnstrasse 3, \\ D-3300 Braunschweig, Federal Republic of Germany
}

\begin{abstract}
Introducing the Hellmann-Weizsäcker functional for large angular momenta and the orbitals of the Bohr atom for small angular momenta we obtain an upper bound on the quantum mechanical ground state energy of atoms that proves Scott's conjecture.
\end{abstract}

\section{Introduction}

Let $H$ be the hamiltonian of $N$ electrons moving in the field of a nucleus of charge $Z$, i.e.

$$
H=\sum_{i=1}^{N}\left(-\Delta_{\imath}-\frac{Z}{\left|\mathbf{r}_{i}\right|}\right)+\sum_{\substack{i, j=1 \\ i<j}}^{N} \frac{1}{\left|\mathbf{r}_{i}-\mathbf{r}_{j}\right|}
$$

as a self-adjoint realization on $\bigwedge_{i=1}^{N}\left(L^{2}\left(\mathbb{R}^{3}\right) \otimes \mathbb{C}^{q}\right)$. In this paper we show for the ground state energy $E_{Q}(Z, N)$ of $H$ :

Theorem 1.1.

$$
E_{Q}(Z, Z) \leqq Z^{7 / 3} E_{1}^{\mathrm{TF}}(1)+\frac{q}{8} Z^{2}+o\left(Z^{2}\right)
$$

where $E_{Z}^{\mathrm{TF}}(N)$ is the Thomas-Fermi energy of the above hamiltonian.

Combining this result with the reverse of (1.1), an inequality that has been claimed by Hughes [1], would imply

$$
E_{Q}(Z, Z)=E_{1}^{\mathrm{TF}}(1) Z^{7 / 3}+\frac{q}{8} Z^{2}+o\left(Z^{2}\right)
$$

Scott [2] claimed this result arguing that the leading energy which is given by the Thomas-Fermi energy (Lieb and Simon [3], Lieb [4], Thirring [5]) should be corrected in those regions where the assumptions of the statistical theory of the

* Supported by the Deutsche Forschungsgemeinschaft 
atom are not well fulfilled. The leading correction turned out to be generated by the electrons near the nucleus where the interaction between the electrons is dominated by the electron-nucleus interaction. Later Scott's conjecture was amended (Lieb [4]) and further supported by heuristic arguments (Schwinger [6]; Englert and Schwinger [7]; Bander [8]).

The strategy of the proof follows Scott's intuition, at least partially: The electrons with high angular momentum are treated statistically via the HellmannWeizsäcker functional $[9,10]$. Only the electrons with small angular momentum whose radial density vanishes only slowly at the origin compared to the bulk of the electrons are treated by introducing Bohr orbitals i.e. orbitals which describe electrons interacting with the nucleus only.

The organization of the paper is as follows: In Sect. two we introduce the basic density matrix and estimate its expectation, in Sect. three we show that we obtain the Hellmann functional plus the $\frac{q}{8} Z^{2}$ correction, and, finally, in the last section we show that the Hellmann functional with our trial density may be bounded from above by the Thomas-Fermi energy.

\section{The Basic One-Particle Density Matrix}

We choose the following one-particle density matrix

$$
\begin{aligned}
& d_{1}\left(x, x^{\prime}\right) \\
& =\sum_{n=-\infty}^{\infty} \sum_{l=0}^{\infty} \sum_{m=-l}^{l} \sum_{s=1}^{q} w_{n, l, m, s} \varphi_{n, l}(r) \overline{\varphi_{n, l}\left(r^{\prime}\right)} \chi_{s}(\sigma) \overline{\chi_{s}\left(\sigma^{\prime}\right)} Y_{l, m}(\omega) \overline{Y_{l, m}\left(\omega^{\prime}\right)},
\end{aligned}
$$

where $x, x^{\prime}$ denote space-spin variables, the weights $w_{n, l, m, s}$ are between zero and one, sum up to at most $Z$ and are independent of $m$ and $s$, and $\chi_{s}(\sigma)=\delta_{s, \sigma}$ are spin functions. Now let $L$ be a sequence of integers tending to infinity as $Z$ tends to infinity. We define $L=\left[Z^{1 / 12}\right]$. Then, for $l \geqq L$ we choose the orbitals $\varphi_{n, l}$ to be Macke orbitals. More explicitly we choose

$$
\varphi_{n, l}(r)=e^{\imath k_{n, l} \hbar_{\zeta_{l}}(r)} \frac{\sqrt{\zeta_{l}^{\prime}(r)}}{r},
$$

where $\zeta_{l}:[0, \infty) \rightarrow[0,1)$, monotone increasing, $\zeta_{l}(0)=0$ and $\zeta_{l}(r) \rightarrow 1$ for $r \rightarrow \infty$, i.e.

$$
\zeta_{l}(r)=\int_{0}^{r} \varrho_{l}(t) d t\left(\int_{0}^{\infty} \varrho_{l}(t) d t\right)^{-1}
$$

with nonnegative $\varrho_{l}$. Moreover we require $\varrho_{l}(0)=0$. The functions $\varrho_{l}$ may be interpreted as radial densities in the angular momentum channel $l$. They are chosen as follows

\section{Definition 2.1.}

1. For sufficiently large $Z$, let $\varrho^{H}=\left(\varrho_{0}^{H}, \varrho_{1}^{H}, \ldots\right)$ be defined by

$$
\varrho_{l}^{H}(r)=\alpha_{l}^{-1 / 2} \frac{1}{r} \sqrt{\left[\lambda^{2}(r)-\left(l+\frac{1}{2}\right)^{2}\right]_{+}} .
$$


with $\lambda(r)=\left(1-a Z^{-1 / 2}\right)^{1 / 3} L_{Z}(r)$ for a certain positive constant a which will be chosen later and $\alpha_{l}=\frac{\pi^{2}}{q^{2}(2 l+1)^{2}}$. The function $L_{Z}$, which is essentially the cubic root of the Thomas-Fermi density, is defined in Appendix $B$.

2. Let $r_{1}(l)$ and $r_{2}(l)$ be the points where $\varrho_{l}^{H}$ becomes zero. Furthermore let $k$ be defined by $k+\frac{1}{2}=\max \left\{\lambda(r) \mid r \in \mathbb{R}^{+}\right\}$, and let $k^{\prime}$ be the greatest integer less than $k$. Then we define for sufficiently large $Z$ : For $l \geqq k^{\prime}$ let $\varrho_{l}(r) \equiv 0$. For $0 \leqq l \leqq k^{\prime}-1$, let

$$
\varrho_{l}(\mathrm{r})= \begin{cases}q(2 l+1) \alpha^{2} r^{2 l+2} & \text { for } 0 \leqq r \leqq x_{1}(l) \\ \varrho_{l}^{H} & \text { for } x_{1}(l) \leqq r \leqq x_{2}(l), \\ q(2 l+1) \beta^{2} e^{-2 \gamma r} & \text { for } x_{2}(l) \leqq r\end{cases}
$$

where the constants $\alpha$ and $\beta$ are chosen such that $\varrho_{l}$ becomes continuous at $x_{1}(l)$ and $x_{2}(l)$, i.e.

$$
\alpha^{2}=\frac{\sqrt{R_{l}\left(x_{1}(l)\right)}}{\pi x_{1}^{2 l+3}(l)}, \quad \beta^{2}=\frac{\sqrt{R_{l}\left(x_{2}(l)\right)}}{\pi x_{2}(l)} e^{2 \gamma x_{2}(l)},
$$

where we introduced the abbreviation $R_{l}(r)=\left[\lambda^{2}(r)-\left(l+\frac{1}{2}\right)^{2}\right]_{+}$. Furthermore we set

$$
\gamma=Z^{2 / 3}, \quad x_{1}(l)=r_{1}(l)+T \frac{l+\frac{1}{2}}{Z}, \quad x_{2}(l)=r_{2}(l)-S Z^{-2 / 3} .
$$

$T$ and $S$ are positive constants. $S$ is arbitrary, while $T$ is in $(0,1)$. For $l \geqq k^{\prime}$ we set $x_{1}(l)=x_{2}(l)$ to be an arbitrary constant.

Next we define the weights $w_{n, l, m, s}$ and the phase factors $k_{n, l}$. Let $N_{l, m, s}=\int_{0}^{\infty} \varrho_{l}(r) d r /(q(2 l+1))$. If $\left[N_{l, m, s}\right]$ is odd, we choose $k_{n, l}=2 n$, otherwise $k_{n, l}=2 n-1$. The weights are chosen as

$$
w_{n, l . m, s}= \begin{cases}1 & \text { for }\left|k_{n, l}\right| \leqq\left[N_{l, m, s}\right]-1 \\ \varepsilon_{l} / 2=\left(N_{l, m, s}-\left[N_{l, m, s}\right]\right) / 2 & \text { for }\left|k_{n, l}\right|=\left[N_{l, m, s}\right]+1 \\ 0 & \text { otherwise. }\end{cases}
$$

This defines the orbitals for high angular momentum completely and yields onedimensional Thomas-Fermi-Weizsäcker functionals in these angular momentum channels. Our choice of the radial densities reflects the fact that we expect the bulk of the electron well described by a "statistical model". Thus we choose them to be approximate minimizing Hellmann densities [11,10]. However, at places where these densities become small, i.e. near the origin and near infinity, we cannot expect this a good approximation. The gradient term would in fact diverge where the radial densities vanish. Thus we modify them in these regions according to the asymptotics of the Bohr atom. Up to this point the ansatz for the one-particle density matrix is exactly the same as in [9]. However, for $l<L$ we choose the $\varphi_{n, l}$ to be the first (weight one) $K-l$ eigenfunction of the radial Schrödinger equation in a Coulomb field with point charge $Z$. We set $K=\left[d Z^{1 / 3}\right]$ with some positive constant $d$. 
Thus the electrons near the nucleus are treated without statistical approximation but with orbitals belonging to non-interacting electrons. For electrons with $l \geqq L$ the interaction is taken into account but only within a statistical model.

The ground state energy may be bounded by using the usual Hartree-Fock functional written as a functional of the one-particle density matrix. Furthermore we estimate the indirect part of the Coulomb energy by zero (positivity of the Coulomb kernel). We remark that this density matrix is admissible for this functional: The weights are given explicitly between zero and one and $d_{1}$ has finite kinetic energy (see [9] and the proofs of Lemma 4.1 and of Proposition 3.3). Because of Theorem 5.1 of [9] it suffices to show that $d_{1}$ represents $Z$ particles or less. To show this trace property we need some more results, and thus we shall come back to this point in chapter four.

Next we calculate the value of this functional explicitly. According to [9] and the fact that the energy levels of the Bohr atom are $-\frac{Z^{2}}{4} \frac{1}{n^{2}}$ in our units, we obtain

$$
\begin{aligned}
E_{Q}(Z, Z) & \leq-\frac{Z^{2}}{4} \sum_{l=0}^{L-1} q(2 l+1) \sum_{n=l+1}^{K} \frac{1}{n^{2}} \\
& +\sum_{l=L}^{\infty} \int_{0}^{\infty} \sqrt{\varrho_{l}^{\prime 2}}+\frac{\alpha_{l}}{3} \varrho_{l}^{3}+\left(\frac{l(l+1)}{r^{2}}-\frac{Z}{r}\right) \varrho_{l} d r \\
& +\frac{1}{2} \int_{0}^{\infty} \int_{0}^{\infty} \sum_{l, l^{\prime}=L}^{\infty} \varrho_{l}(r) \varrho_{l^{\prime}}\left(r^{\prime}\right) \frac{1}{\max \left\{r, r^{\prime}\right\}} d r^{\prime} d r \\
& +\frac{1}{2} \int_{0}^{\infty} \int_{0}^{\infty}\left(2 \sum_{l=0}^{L-1} q(2 l+1) \sum_{n=l+1}^{K} \varphi_{n, l}^{2}(r) \sum_{l^{\prime}=L}^{\infty} \varrho_{l^{\prime}}\left(r^{\prime}\right)\right. \\
& \left.+\sum_{l, l^{\prime}=0}^{L-1} q(2 l+1) q\left(2 l^{\prime}+1\right) \sum_{n=l+1}^{K} \sum_{n^{\prime}=l^{\prime}+1}^{K} \varphi_{n, l}^{2}(r) \varphi_{n^{\prime}, l^{\prime}}^{2}\left(r^{\prime}\right)\right) \frac{1}{\max \left\{r, r^{\prime}\right\}} d r^{\prime} d r \\
& +\sum_{l=L}^{\infty} \frac{\alpha_{l}}{3}\left(\frac{-1+6 \varepsilon_{l}-3 \varepsilon_{l}^{2}}{N_{l, m, s}^{2}}+\frac{2 \varepsilon_{l}^{3}-6 \varepsilon_{l}^{2}+4 \varepsilon_{l}}{N_{l, m, s}^{3}}\right) \int_{0}^{\infty} \varrho_{l}^{3} d r,
\end{aligned}
$$

a result that follows from Theorem 5.1 of [9]. It is based on Lieb's upper bound on the ground state energy [12].

\section{The Hellmann Functional Plus $\frac{q}{8} Z^{2}$ Bounds the Ground State Energy from Above}

We begin this chapter with some definitions: Denote by $E_{l, Z}^{H}\left(N_{l}\right)$ the infimum of the functional

$$
\mathscr{E}_{l, Z}^{H}\left(\sigma_{l}\right)=\int_{0}^{\infty}\left(\frac{1}{3} \alpha_{l} \sigma_{l}^{3}+\left(\frac{\left(l+\frac{1}{2}\right)^{2}}{r^{2}}-\frac{Z}{r}\right) \sigma_{l}\right) d r
$$


taken over the densities with particle number $N_{l}$ or less. Analogously we define $E_{l, Z}^{H W}\left(N_{l}\right)$ as the infimum of

$$
\mathscr{E}_{l, Z} H\left(\sigma_{l}\right)=\int_{0}^{\infty}\left(\sqrt{\sigma_{l}{ }^{2}}+\frac{\alpha_{l}}{3} \sigma_{l}^{3}+\left(\frac{l(l+1)}{r^{2}}-\frac{Z}{r}\right) \sigma_{l}\right) d r .
$$

Finally we introduce the functional $\mathscr{E}_{Z}^{H}(\underline{\sigma})$ to be the sum over all non-negative integers $l$ of $\mathscr{E}_{l, Z}^{H}\left(\sigma_{l}\right)$ plus $\frac{1}{2} \sum_{l, l^{\prime}=0}^{\infty} \int_{0}^{\infty} d r \int_{0}^{\infty} d r^{\prime} \frac{\sigma_{l}(r) \sigma_{l^{\prime}}\left(r^{\prime}\right)}{\max \left\{r, r^{\prime}\right\}}$. (For a detailed investigation of these functionals see [9].)

In this chapter we shall prove the

Lemma 3.1. $E_{Q}(Z, Z) \leqq \mathscr{E}_{Z}^{H}\left(\varrho^{H}\right)+\frac{q}{8} Z^{2}+O\left(Z^{47 / 24}\right)$.

To this end we collect some technical results:

\section{Proposition 3.1.}

$$
\frac{-Z^{2}}{4} \sum_{l=0}^{L-1} q(2 l+1) \sum_{n=l+1}^{K} \frac{1}{n^{2}}=\frac{q}{8} Z^{2}+\sum_{l=0}^{L-1} E_{l, Z}^{H}(q(2 l+1)(K-l))+O\left(Z^{23 / 12}\right) .
$$

Proof. In order to evaluate the sum over $n$ we use the following asymptotic expansion as $x \rightarrow \infty$ of the Riemann zeta function (see Erdelyi et al. [13], formula 1.18(9)):

$$
\zeta(s, x)=\sum_{n=0}^{\infty}(n+x)^{-s}=\frac{\Gamma(s-1)}{\Gamma(s)} x^{1-s}+\frac{1}{2} x^{-s}+O\left(x^{-1-s}\right) .
$$

Rearranging the sums over $n$ and $l$ and using (3.1), we find after some algebra,

$$
\sum_{l=0}^{L-1} \sum_{n=l+1}^{K} \frac{2 l+1}{n^{2}}=2 L-\frac{1}{2}+O\left(L^{-1}\right)
$$

for our choice of $L$ and $K$. In [11], Eq. 2.9, we determined $E_{l, Z}^{H}(q(2 l+1)(K-l))$ to be $\frac{-Z^{2} q}{4} \frac{4(K-l)}{2 K+1}$. We find

$$
\sum_{l=0}^{L-1} \frac{4(K-l)}{2 K+1}=2 L-\frac{2 L^{2}}{2 K+1} .
$$

The equalities (3.2) and (3.3) yield

$$
\sum_{l=0}^{L-1} \sum_{n=l+1}^{K} \frac{2 l+1}{n^{2}}=-\frac{1}{2}+\sum_{l=0}^{L-1} \frac{4(K-l)}{2 K+1}+O\left(L^{-1}\right) .
$$

Multiplication with $\frac{-Z^{2} q}{4}$ yields the proposed result.

Proposition 3.2. There exists a positive constant $d$ such that for our choice of $K$, namely $K=\left[d Z^{1 / 3}\right]$, and for sufficiently large $Z$

$$
E_{l, Z}^{H}(q(2 l+1)(K-l)) \leqq \mathscr{E}_{l, Z}^{H}\left(\varrho_{l}^{H}\right)
$$


Proof. Since $E_{l, Z}^{H}$ denotes the infimum of $\mathscr{E}_{l, Z}^{H}$, we have only to prove that

$$
\int_{0}^{\infty} \varrho_{l}^{H}(r) d r \leqq q(2 l+1)(K-l) \text {. }
$$
Since $\lambda$ may be enlarged either by $\sqrt{Z r}$ or by $\frac{18 \pi}{q r}$ we find for some positive
constant $c$

$$
\int_{0}^{\infty} \varrho_{l}^{H} d r \leqq \frac{q(2 l+1)}{\pi}\left(\int_{r_{1}(l)}^{R Z^{-1 / 3}} \frac{\sqrt{Z r}}{r} d r+\int_{R Z^{-1 / 3}}^{r_{2}(l)} \frac{18 \pi}{q r^{2}} d r\right) \leqq c q(2 l+1) Z^{1 / 3} .
$$

Hence the claim follows, if we choose, for instance, $d=c+1$.

Proposition 3.3. For sufficiently large $Z$ the following holds:

$$
\sum_{l=L}^{\infty} \mathscr{E}_{l, Z}^{H W}\left(\varrho_{l}\right) \leqq \sum_{l=L}^{\infty} \mathscr{E}_{l, Z}^{H}\left(\varrho_{l}^{H}\right)+O\left(Z^{47 / 24}\right) .
$$

Proof. We remark that each $R_{l}$ has exactly one maximum at $R Z^{-1 / 3}$, since $\lambda$ has a single maximum at $R Z^{-1 / 3}$, and that $\lambda^{\prime \prime}$ has only one zero at $W Z^{-1 / 3}$ for some positive constants $R$ and $W$. These facts follow from Appendix A. Throughout the following we need the results of Lemma C1.

By integration we obtain

$$
\begin{gathered}
\int_{0}^{x_{1}(l)}\left(\sqrt{\varrho_{l}}{ }^{\prime 2}+\frac{\alpha_{l}}{3} \varrho_{l}^{3}+\left(\frac{\left(l+\frac{1}{2}\right)^{2}}{r^{2}}-\frac{Z}{r}\right) \varrho_{l}\right) d r \leqq \operatorname{const} Z^{2}\left(l+\frac{1}{2}\right)^{-3 / 2}, \\
\int_{x_{2}(l)}^{\infty}\left({\sqrt{\varrho_{l}}}^{\prime 2}+\frac{\alpha_{l}}{3} \varrho_{l}^{3}+\left(\frac{\left(l+\frac{1}{2}\right)^{2}}{r^{2}}-\frac{Z}{r}\right) \varrho_{l}\right) d r \leqq \operatorname{const} Z^{7 / 6}\left(l+\frac{1}{2}\right) .
\end{gathered}
$$

Next we consider the gradient term in the middle region. First we calculate the integrand for $0 \leqq l \leqq k^{\prime}-1$ and $x_{1}(l) \leqq r \leqq x_{2}(l)$, and get:

$$
{\sqrt{\varrho_{l}}}^{\prime 2}=\frac{q(2 l+1)}{4 \pi}\left[\frac{\lambda^{2} \lambda^{\prime 2}}{r \sqrt{\lambda^{2}-\left(l+\frac{1}{2}\right)^{2}}}-2 \frac{\lambda \lambda^{\prime}}{r^{2} \sqrt{\lambda^{2}-\left(l+\frac{1}{2}\right)^{2}}}+\frac{\sqrt{\lambda^{2}-\left(l+\frac{1}{2}\right)^{2}}}{r^{3}}\right] .
$$

Now we estimate the integrals. Since $\sqrt{\lambda^{2}-\left(l+\frac{1}{2}\right)^{2}} \leqq \lambda$, we find

$$
\int_{x_{1}(l)}^{x_{2}(l)} \frac{\sqrt{\lambda^{2}-\left(l+\frac{1}{2}\right)^{2}}}{r^{3}} d r \leqq \frac{2}{3} Z^{2}\left(l+\frac{1}{2}\right)^{-3} .
$$

The second term of the sum is negative, if $r \in\left[x_{1}(l), R Z^{-1 / 3}\right]$. Therefore we consider only the interval $\left[R Z^{-1 / 3}, x_{2}(l)\right]$. Using the substitution $\lambda^{2}(r)=x$ we find:

$$
\int_{R Z^{-1 / 3}}^{x_{2}(l)} \frac{-2 \lambda \lambda^{\prime}}{r^{2} \sqrt{\lambda^{2}-\left(l+\frac{1}{2}\right)^{2}}} d r \leqq \frac{2}{R^{2}} Z^{2 / 3}\left(k+\frac{1}{2}\right) \leqq \text { const } Z .
$$

The first term of the sum may be divided into two parts by splitting the integration interval at $R Z^{-1 / 3}$. Considering the first part yields, using the substitution $\lambda(r)=x$,

$$
\int_{x_{1}(l)}^{R Z^{-1 / 3}} \frac{\lambda^{2} \lambda^{\prime 2}}{r \sqrt{\lambda^{2}-\left(l+\frac{1}{2}\right)^{2}}} d r \leqq Z \lambda^{\prime}\left(x_{1}(l)\right) \int_{\lambda\left(x_{1}(l)\right)}^{\lambda\left(R Z^{-1 / 3}\right)} \frac{d x}{\sqrt{x^{2}-\left(l+\frac{1}{2}\right)^{2}}},
$$


since $\frac{x^{2}}{Z} \leqq r$. By the mean value theorem we get $\lambda^{\prime}\left(x_{1}(l)\right) \leqq \frac{\lambda\left(x_{1}(l)\right)-\left(l+\frac{1}{2}\right)}{x_{1}(l)-r_{1}(l)}$. Carrying out the integration yields the estimate $\frac{Z^{2} \sqrt{\lambda^{2}\left(x_{1}(l)\right)-\left(l+\frac{1}{2}\right)^{2}}}{T\left(l+\frac{1}{2}\right)^{3}}$. Hence
we obtain

$$
\int_{x_{1}(l)}^{R Z^{-1 / 3}} \frac{\lambda^{2} \lambda^{\prime 2}}{r \sqrt{\lambda^{2}-\left(l+\frac{1}{2}\right)^{2}}} d r \leqq \text { const } Z^{2}\left(l+\frac{1}{2}\right)^{-5 / 2} \text {. }
$$

The integral $\int_{R Z^{-1 / 3}}^{x_{2}(l)} \frac{\lambda^{2} \lambda^{\prime 2}}{r \sqrt{\lambda^{2}-\left(l+\frac{1}{2}\right)^{2}}} d r$ remains. We enlarge the integrand using $-\frac{\lambda(r) \lambda^{\prime}(r)}{r} \leqq$ const $Z^{4 / 3}$, since $\lambda^{\prime}(r) \geqq-$ const $Z^{2 / 3}$ holds. Hence

$$
\begin{aligned}
\int_{R Z^{-1 / 3}}^{x_{2}(l)} \frac{\lambda^{2} \lambda^{\prime 2} d r}{r \sqrt{\lambda^{2}-\left(l+\frac{1}{2}\right)^{2}}} & \leqq \text { const } Z^{4 / 3} \int_{\lambda^{2}\left(x_{2}(l)\right)}^{\lambda^{2}\left(R Z^{-1 / 3}\right)} \frac{d x}{\sqrt{x-\left(l+\frac{1}{2}\right)^{2}}} \\
& \leqq \frac{\operatorname{const} Z^{5 / 3}}{\left(l+\frac{1}{2}\right)^{1 / 2} \min \left\{l+\frac{1}{2}, Z^{1 / 4}\right\}} .
\end{aligned}
$$

Combining the results $(3.5-3.8)$ yields

$$
\begin{aligned}
& \int_{x_{1}(l)}^{x_{2}(l)}{\sqrt{\varrho_{l}}}^{\prime 2} d r \leqq \text { const }\left(l+\frac{1}{2}\right) \\
& \cdot\left[Z^{2}\left(l+\frac{1}{2}\right)^{-3}+Z+Z^{2}\left(l+\frac{1}{2}\right)^{-5 / 2}+\frac{Z^{5 / 3}\left(l+\frac{1}{2}\right)^{-1 / 2}}{\min \left\{l+\frac{1}{2}, Z^{1 / 4}\right\}}\right] .
\end{aligned}
$$

Since for $0 \leqq l \leqq k^{\prime}-1$,

$$
\begin{aligned}
& \int_{r_{1}(l)}^{x_{1}(l)} \frac{Z}{r} \varrho_{l}^{H}(r) d r \leqq \frac{q(2 l+1)}{\pi} Z \sqrt{R_{l}\left(x_{1}(l)\right)} \frac{x_{1}(l)-r_{1}(l)}{x_{1}(l) r_{1}(l)} \leqq \operatorname{const} Z^{2}\left(l+\frac{1}{2}\right)^{-3 / 2}, \\
& \int_{x_{2}(l)}^{r_{2}(l)} \frac{Z}{r} \varrho_{l}^{H}(r) d r \leqq \frac{q(2 l+1)}{\pi} Z \sqrt{R_{l}\left(x_{2}(l)\right)} \frac{r_{2}(l)-x_{2}(l)}{x_{2}(l) r_{2}(l)} \leqq \operatorname{const} Z^{7 / 6}\left(l+\frac{1}{2}\right)
\end{aligned}
$$

holds, we have

$$
\int_{x_{1}(l)}^{x_{2}(l)}\left(\frac{\alpha_{l}}{3} \varrho_{l}^{3}+\left(\frac{l(l+1)}{r^{2}}-\frac{Z}{r}\right) \varrho_{l}\right) d r \leqq \mathscr{E}_{l, Z}^{H}\left(\varrho_{l}^{H}\right)+\operatorname{const} Z^{2}\left(l+\frac{1}{2}\right)^{-3 / 2} .
$$

Moreover for $l=k^{\prime}$,

$$
\int_{r_{1}\left(k^{\prime}\right)}^{r_{2}\left(k^{\prime}\right)} \frac{Z}{r} \varrho_{k^{\prime}}^{H}(r) d r \leqq \operatorname{const} Z^{11 / 6}
$$

holds. 
Hence we have from (3.4) and (3.9-3.11),

$$
\begin{aligned}
& \sum_{l=L}^{\infty} \mathscr{E}_{l, Z}^{H W}\left(\varrho_{l}\right) \leqq \sum_{l=L}^{\infty} \mathscr{E}_{l, Z}^{H}\left(\varrho_{l}^{H}\right) \\
& \quad+\text { const } \sum_{l=L}^{k^{\prime}-1}\left(Z^{2}\left(l+\frac{1}{2}\right)^{-3 / 2}+\frac{Z^{5 / 3}\left(l+\frac{1}{2}\right)^{1 / 2}}{\min \left\{l+\frac{1}{2}, Z^{1 / 4}\right\}}\right)+O\left(Z^{11 / 6}\right) .
\end{aligned}
$$

Using (3.1) we get that $\sum_{l=L}^{k^{\prime}-1} Z^{2}\left(l+\frac{1}{2}\right)^{-3 / 2}=O\left(Z^{2} L^{-1 / 2}\right)$. The remaining sum yields a term of order $Z^{23 / 12}$. This completes the proof.

Now we consider the electron-electron interaction.

\section{Proposition 3.4.}

$$
\sum_{l, l^{\prime}=0}^{\infty} \int_{0}^{\infty} \int_{0}^{\infty} \frac{\varrho_{l}(r) \varrho_{l^{\prime}}\left(r^{\prime}\right)}{\max \left\{r, r^{\prime}\right\}} d r^{\prime} d r \leqq \sum_{l, l=0}^{\infty} \int_{0}^{\infty} \int_{0}^{\infty} \frac{\varrho_{l}^{H}(r) \varrho_{l^{\prime}}^{H}\left(r^{\prime}\right)}{\max \left\{r, r^{\prime}\right\}} d r^{\prime} d r+\operatorname{const} Z^{11 / 6} .
$$

Proof. We divide the interaction integral into several parts:

$$
\begin{aligned}
& \int_{0}^{\infty} \int_{0}^{\infty} \frac{\varrho_{l}(r) \varrho_{l^{\prime}}\left(r^{\prime}\right)}{\max \left\{r, r^{\prime}\right\}} d r^{\prime} d r \leqq \int_{0}^{x_{1}(l)} \varrho_{l}(r) d r \int_{0}^{x_{1}\left(l^{\prime}\right)} \frac{\varrho_{l^{\prime}}\left(r^{\prime}\right)}{r^{\prime}} d r^{\prime} \\
& \quad+\int_{0}^{x_{1}(l)} \varrho_{l}(r) d r \int_{x_{1}\left(l^{\prime}\right)}^{x_{2}\left(l^{\prime}\right)} \frac{\varrho_{l^{\prime}}\left(r^{\prime}\right)}{r^{\prime}} d r^{\prime}+\int_{0}^{x_{1}(l)} \varrho_{l}(r) d r \int_{x_{2}\left(l^{\prime}\right)}^{\infty} \frac{\varrho_{l^{\prime}}\left(r^{\prime}\right)}{r^{\prime}} d r^{\prime} \\
& \quad+\int_{x_{1}(l)}^{x_{2}(l)} \frac{\varrho_{l}(r)}{r} d r \int_{0}^{x_{1}\left(l^{\prime}\right)} \varrho_{l^{\prime}}\left(r^{\prime}\right) d r^{\prime}+\int_{x_{1}(l)}^{x_{2}(l)} \int_{x_{1}\left(l^{\prime}\right)}^{x_{2}\left(l^{\prime}\right)} \frac{\varrho_{l}(r) \varrho_{l^{\prime}}\left(r^{\prime}\right)}{\max \left\{r, r^{\prime}\right\}} d r^{\prime} d r \\
& +\int_{x_{1}(l)}^{x_{2}(l)} \varrho_{l}(r) d r \int_{x_{2}\left(l^{\prime}\right)}^{\infty} \frac{\varrho_{l^{\prime}}\left(r^{\prime}\right)}{r^{\prime}} d r^{\prime}+\int_{x_{2}(l)}^{\infty} \frac{\varrho_{l}(r)}{r} d r \int_{0}^{x_{1}\left(l^{\prime}\right)} \varrho_{l^{\prime}}\left(r^{\prime}\right) d r^{\prime} \\
& \left.\quad+\int_{x_{2}(l)}^{\infty} \frac{\varrho_{l}(r)}{r} d r \int_{x_{1}\left(l^{\prime}\right)}^{x_{2}\left(l^{\prime}\right)} \varrho_{l^{\prime}}\left(r^{\prime}\right) d r^{\prime}+\int_{x_{2}\left(l^{\prime}\right)}^{\infty} \frac{\varrho_{l}(r)}{r} d r \int_{l^{\prime}}^{\infty} r^{\prime}\right) d r^{\prime},
\end{aligned}
$$

since we may enlarge $\frac{1}{\max \left\{r, r^{\prime}\right\}}$ either by $\frac{1}{r}$ or by $\frac{1}{r^{\prime}}$. Using Lemma C.1 we calculate the following estimates:

$$
\begin{aligned}
& \sum_{l=0}^{\infty} \int_{0}^{x_{1}(l)} \varrho_{l} d r \leqq \text { const } Z^{1 / 2}, \\
& \sum_{l=0}^{\infty} \int_{x_{1}(l)}^{x_{2}(l)} \varrho_{l} d r \leqq \text { const } Z, \\
& \sum_{l=0}^{\infty} \int_{x_{2}(l)}^{\infty} \varrho_{l} d r \leqq \text { const } Z^{1 / 2}, \\
& \sum_{l=0}^{\infty} \int_{0}^{x_{1}(l)} \frac{\varrho_{l}}{r} d r \leqq \text { const } Z, \\
& \sum_{l=0}^{\infty} \int_{x_{1}(l)}^{x_{2}(l)} \frac{\varrho_{l}}{r} d r \leqq \text { const } Z^{4 / 3}, \\
& \sum_{l=0}^{\infty} \int_{x_{2}(l)}^{\infty} \frac{\varrho_{l}}{r} d r \leqq \text { const } Z^{5 / 6},
\end{aligned}
$$

where we used $R_{l}(r) \leqq Z r$ for the fifth integral. This yields the proposed result. 


\section{Proposition 3.5.}

$$
\begin{aligned}
& \frac{1}{2} \sum_{l, l^{\prime}=0}^{L-1} \int_{0}^{\infty} \int_{0}^{\infty} \sum_{n=l+1}^{K} q(2 l+1) \varphi_{n, l}^{2}(r) \sum_{n^{\prime}=l^{\prime}+1}^{K} q\left(2 l^{\prime}+1\right) \varphi_{n^{\prime}, l^{\prime}}^{2}\left(r^{\prime}\right) \frac{1}{\max \left\{r, r^{\prime}\right\}} d r^{\prime} d r \\
& \quad+\sum_{l=0}^{L-1} \sum_{l^{\prime}=L}^{\infty} \int_{0}^{\infty} \int_{0}^{\infty} \sum_{n=l+1}^{K} q(2 l+1) \varphi_{n, l}^{2}(r) \varrho_{l^{\prime}}\left(r^{\prime}\right) \frac{1}{\max \left\{r, r^{\prime}\right\}} d r^{\prime} d r \leqq \operatorname{const} Z^{11 / 6} .
\end{aligned}
$$

Proof. We use $\frac{1}{\max \left\{r, r^{\prime}\right\}} \leqq \frac{1}{r^{\prime}}$. Then we estimate the first integral using the virial theorem for the hamiltonian of the Bohr atom by

$$
\sum_{l, l^{\prime}=0}^{L-1} q(2 l+1)(K-l) \sum_{n^{\prime}=l^{\prime}+1}^{K} q\left(2 l^{\prime}+1\right) \frac{Z}{2 n^{\prime 2}} \leqq \text { const } Z K L^{3} .
$$

In the proof of Proposition 3.4 we showed that $\sum_{l=0}^{\infty} \int_{0}^{\infty} \frac{\varrho_{l}(r)}{r} d r \leqq$ const $Z^{4 / 3}$. Hence we find for the second integral the upper bound,

$$
\text { const } Z^{4 / 3} \sum_{l=0}^{L-1} q(2 l+1)(K-l) \leqq \text { const } Z^{4 / 3} K L^{2} \text {. }
$$

This shows the desired result.

\section{Proposition 3.6.}

$$
\sum_{\substack{l=0 \\ N_{l, m, s} \neq 0}}^{\infty} \frac{\alpha_{l}}{3}\left(\frac{-1+6 \varepsilon_{l}-3 \varepsilon_{l}^{2}}{N_{l, m, s}^{2}}+\frac{2 \varepsilon_{l}^{3}-6 \varepsilon_{l}^{2}+4 \varepsilon_{l}}{N_{l, m, s}^{3}}\right) \int_{0}^{\infty} \varrho_{l}^{3} d r \leqq \operatorname{const} Z^{5 / 3}
$$

Proof. Let $\varrho_{l, m, s}=\varrho_{l} /(q(2 l+1))$. Then we shall show in the following that

$$
\int_{0}^{\infty} \varrho_{l, m, s}^{3} d r \leqq \text { const } Z^{4 / 3} \frac{(k-l)^{2}}{l+\frac{1}{2}},
$$

and

$$
N_{l, m, s} \geqq \operatorname{const}(k-l) .
$$

This proves the proposition.

We may write:

$$
\int_{x_{1}(l)}^{x_{2}(l)} \varrho_{l, m, s}^{3} d r \geqq \frac{\sqrt{R_{l}^{3}\left(x_{1}(l)\right)}}{2 \pi^{3} x_{1}(l)^{2}}\left(1-\frac{x_{1}(l)}{R Z^{-1 / 3}}\right) .
$$

If $x_{1}(l) \leqq R_{0} Z^{-1 / 3}$ with $R_{0}<R$, then $(6 l+7)\left(1-\frac{x_{1}(l)}{R Z^{-1 / 3}}\right) \geqq$ const. Otherwise, if $x_{1}(l)>R_{0} Z^{-1 / 3}$, then $\left(l+\frac{1}{2}\right) \geqq$ const $Z^{1 / 3}$ by Lemma C.1. This yields $(6 l+7)\left(1-\frac{x_{1}(l)}{R Z^{-1 / 3}}\right) \geqq$ const, too. Hence we have

$$
\int_{0}^{x_{1}(l)} \varrho_{l, m, s}^{3} d r \leqq \text { const } \int_{x_{1}(l)}^{x_{2}(l)} \varrho_{l, m, s}^{3} d r
$$


Analogously we find $\int_{x_{1}(l)}^{x_{2}(l)} \varrho_{l, m, s}^{3} d r \geqq$ const $Z^{-1 / 2} \frac{\sqrt{R_{l}^{3}\left(x_{2}(l)\right)}}{\pi^{3} x_{2}(l)^{3}}$, and hence

$$
\int_{x_{2}(l)}^{\infty} \varrho_{l, m, s}^{3} d r \leqq \mathrm{const} \int_{x_{1}(l)}^{x_{2}(l)} \varrho_{l, m, s}^{3} d r
$$

To calculate an estimate for $\int_{x_{1}(l)}^{x_{2}(l)} \varrho_{l, m, s}^{3} d r$ we distinguish two cases:

First Case. $\left(l+\frac{1}{2}\right) \leqq \frac{1}{2}\left(k+\frac{1}{2}\right)$.

We find

$$
\int_{x_{1}(l)}^{x_{2}(l)} \varrho_{l, m, s}^{3} d r \leqq \int_{x_{1}(l)}^{x_{2}(l)} \frac{(Z r)^{3 / 2}}{\pi^{3} r^{3}} d r \leqq \operatorname{const} \frac{Z^{2}}{\left(l+\frac{1}{2}\right)} \leqq \operatorname{const} Z^{4 / 3} \frac{(k-l)^{2}}{\left(l+\frac{1}{2}\right)} .
$$

Second Case. $\left(l+\frac{1}{2}\right) \geqq \frac{1}{2}\left(k+\frac{1}{2}\right)$.

We remark that there are positive constants $R_{1}$ and $R_{2}$ such that $\left[r_{1}(l), r_{2}(l)\right]$ is contained in $\left[R_{1} Z^{-1 / 3}, R_{2} Z^{-1 / 3}\right]$. Choose $R^{\prime}, R^{\prime \prime}$ with $R^{\prime}<R<R^{\prime \prime}<W$. For $r Z^{1 / 3} \in\left[R_{1}, R^{\prime}\right]$ or $r Z^{1 / 3} \in\left[R^{\prime \prime}, R_{2}\right]$ we find $\left|\lambda^{\prime}(r)\right| \geqq$ const $Z^{2 / 3} \geqq$ const $Z^{1 / 2}$ - $\sqrt{k+\frac{1}{2}-\lambda(r)}$ for sufficiently large $Z$ using the scaling property of $L_{Z}^{\prime}$. For $r Z^{1 / 3} \in\left[R^{\prime}, R^{\prime \prime}\right]$ we find certain constants $c_{1}$ and $c_{2}$ with $-c_{1} Z \leqq \lambda^{\prime \prime}(r) \leqq-c_{2} Z$ using again scaling and sufficiently large $Z$. Then, by the mean value theorem, $\left|\lambda^{\prime}(r)\right|=-\left|r-R Z^{-1 / 3}\right| \lambda^{\prime \prime}\left(r^{\prime}\right) \geqq c_{2} Z\left|r-R Z^{-1 / 3}\right|$ for $r^{\prime} \in\left[R^{\prime}, R^{\prime \prime}\right]$. Moreover $\lambda(r)=k+\frac{1}{2}+\frac{1}{2}\left(r-R Z^{-1 / 3}\right)^{2} \lambda^{\prime \prime}\left(r^{\prime \prime}\right)$ for $r, r^{\prime \prime} \in\left[R^{\prime}, R^{\prime \prime}\right]$. Hence

$$
\left|r-R Z^{-1 / 3}\right|=\sqrt{2 \frac{k+\frac{1}{2}-\lambda(r)}{-\lambda^{\prime \prime}\left(r^{\prime \prime}\right)}} \geqq \text { const } Z^{-1 / 2} \sqrt{k+\frac{1}{2}-\lambda(r)} .
$$

Therefore we have for any $r \in\left[R_{1} Z^{-1 / 3}, R_{2} Z^{-1 / 3}\right]$,

$$
\left|\lambda^{\prime}(r)\right| \geqq \text { const } Z^{1 / 2} \sqrt{k+\frac{1}{2}-\lambda(r)} .
$$

Now we can estimate $\int_{x_{1}(l)}^{x_{2}(l)} \varrho_{l, m, s}^{3} d r$ in the following way:

$$
\begin{aligned}
\int_{x_{1}(l)}^{x_{2}(l)} \varrho_{l, m, s}^{3} d r & \leqq \int_{r_{1}(l)}^{R Z^{-1 / 3}} \frac{\sqrt{\lambda^{2}(r)-\left(l+\frac{1}{2}\right)^{2}}}{\pi^{3} r^{3}\left|\lambda^{\prime}(r)\right|} \lambda^{\prime}(r) d r+\int_{r_{2}(l)}^{R Z^{-1 / 3}} \frac{\sqrt{\lambda^{2}(r)-\left(l+\frac{1}{2}\right)^{2}}}{\pi^{3} r^{3}\left|\lambda^{\prime}(r)\right|} \lambda^{\prime}(r) d r \\
& \leqq \operatorname{const} Z^{1 / 2} \int_{l+1 / 2}^{k+1 / 2} \frac{\sqrt{x^{2}-\left(l+\frac{1}{2}\right)^{2}}}{\sqrt{k+\frac{1}{2}-x}} d x \\
& \leqq \text { const } Z \int_{l+1 / 2}^{k+1 / 2} \sqrt{\frac{\left(x-\left(l+\frac{1}{2}\right)\right)^{3}}{k+\frac{1}{2}-x}} d x \leqq \operatorname{const} Z^{4 / 3} \frac{(k-l)^{2}}{l+\frac{1}{2}} .
\end{aligned}
$$

This proves the inequality (3.12). 
Now we estimate $N_{l, m, s}$ :

$$
N_{l, m, s}=\int_{0}^{\infty} \varrho_{l, m, s} d r \geqq \int_{x_{1}(l)}^{R Z^{-1 / 3}} \frac{\sqrt{\lambda^{2}(r)-\left(l+\frac{1}{2}\right)^{2}}}{\pi r L_{Z}^{\prime}(r)} \lambda^{\prime}(r) d r .
$$

First Case. $l+\frac{1}{2} \leqq \frac{1}{2}\left(k+\frac{1}{2}\right)$.

By scaling $r L_{Z}^{\prime}(r) \leqq$ const $Z^{1 / 3}$ holds, since $x \hat{L}^{\prime}(x)$ is bounded. Because $\sqrt{x^{2}-\left(l+\frac{1}{2}\right)^{2}} \geqq x-\frac{1}{2}\left(l+\frac{1}{2}\right)-\frac{1}{2}\left(l+\frac{1}{2}\right)^{4} x^{-3}$ for $x \geqq l+\frac{1}{2}$, we find by substituting $\lambda(r)=x$,

$$
N_{l, m, s} \geqq \operatorname{const} Z^{-1 / 3}(k-l)\left(\frac{3}{8}\left(k+\frac{1}{2}\right)-\frac{T}{2}\right) \geqq \operatorname{const}(k-l) .
$$

Second Case. $l+\frac{1}{2} \geqq \frac{1}{2}\left(k+\frac{1}{2}\right)$.

In this case we know that $x_{1}(l) \geqq R_{1} Z^{-1 / 3}$. We now show in a similar way as in the proof of (3.14) that $\lambda^{\prime}(r) \leqq$ const $Z^{1 / 2} \sqrt{k+\frac{1}{2}-\lambda(r)}$. Hence

$$
\begin{aligned}
N_{l, m, s} & \geqq \operatorname{const} Z^{-1 / 6} \int_{x_{1}(l)}^{R Z^{-1 / 3}} \sqrt{\frac{\lambda^{2}(r)-\left(l+\frac{1}{2}\right)^{2}}{k+\frac{1}{2}-\lambda(r)}} \lambda^{\prime}(r) d r \\
& \geqq \text { const } \int_{0}^{\sqrt{k+\frac{1}{2}-\lambda\left(x_{1}(l)\right)}} \sqrt{k-l-x^{2}} d x \\
& \geqq \operatorname{const}(k-l) \arcsin \sqrt{1-\frac{T}{k-l}} \geqq \operatorname{const}(k-l)
\end{aligned}
$$

for $T<1$. This shows inequality (3.13) and completes the proof.

\section{Estimate for $\mathscr{E}_{Z}^{H}\left(\varrho^{H}\right)$ by the Thomas-Fermi Energy}

In this chapter we prove:

Lemma 4.1. Let $\underline{Q}^{I I}$ be defined as in (2.2). Then

$$
\mathscr{E}_{Z}^{H}\left(\underline{\varrho}^{H}\right) \leqq E_{Z}^{\mathrm{TF}}(Z)+O\left(Z^{5 / 3}\right) .
$$

First we calculate $\sum_{l=0}^{\infty} \varrho_{l}^{H}(r)$ and $\sum_{l=0}^{\infty}\left(\frac{\alpha_{l}}{3}\left(\varrho_{l}^{H}\right)^{3}(r)+\frac{\left(l+\frac{1}{2}\right)^{2}}{r^{2}} \varrho_{l}^{H}(r)\right)$ following an idea of Englert and Schwinger [14] using Poisson's summation formula (see e.g. Bochner [15]) which states

$$
\sum_{l=-\infty}^{\infty} F\left(l+\frac{1}{2}\right)=\sum_{n=-\infty}^{\infty} e^{-\pi i n} \int_{-\infty}^{\infty} e^{2 \pi i n x} F(x) d x
$$


where the sum is meant as principal value $\left(\sum_{i=-\infty}^{\infty} a_{i}=\lim _{n \rightarrow \infty} \sum_{i=-n}^{n} a_{i}\right)$. Therefore we
have:

$$
\begin{gathered}
\sum_{l=0}^{\infty} \varrho_{l}^{H}(r)=\sum_{n=-\infty}^{\infty}(-1)^{n} \frac{2 q}{\pi r} \int_{0}^{\lambda} e^{2 \pi i n x} x \sqrt{\lambda^{2}-x^{2}} d x \\
\sum_{l=0}^{\infty} \frac{\alpha_{l}}{3}\left(\varrho_{l}^{H}\right)^{3}(r)=\sum_{n=-\infty}^{\infty}(-1)^{n} \frac{2 q}{3 \pi r^{3}} \int_{0}^{\lambda} e^{2 \pi i n x} x \sqrt{\lambda^{2}-x^{2}} d x \\
\sum_{l=0}^{\infty} \frac{\left(l+\frac{1}{2}\right)^{2}}{r^{2}} \varrho_{l}^{H}(r)=\sum_{n=-\infty}^{\infty}(-1)^{n} \frac{2 q}{\pi r^{3}} \int_{0}^{\lambda} e^{2 \pi i n x} x^{3} \sqrt{\lambda^{2}-x^{2}} d x
\end{gathered}
$$

We may combine the terms $n$ and $-n$. The integrals are then solved after an integration by parts by Struve functions $\mathbf{H}_{v}$ yielding

$$
\begin{aligned}
& \sum_{l=0}^{\infty} \varrho_{l}^{H}=\frac{2 q}{3 \pi r} \lambda^{3}+\frac{q}{r} \lambda^{3} \sum_{n=1}^{\infty}(-1)^{n}\left(\frac{4}{3 \pi}-\frac{\mathbf{H}_{2}(2 \pi n \lambda)}{\pi n \lambda}\right), \\
& \sum_{l=0}^{\infty}\left(\frac{\alpha_{l}}{3}\left(\varrho_{l}^{H}\right)^{3}+\frac{\left(l+\frac{1}{2}\right)^{2}}{r^{2}} \varrho_{l}^{H}\right) \\
& \quad=\frac{2 q}{5 \pi r^{3}} \lambda^{5}+\frac{q}{r^{3}} \lambda^{5} \sum_{n=1}^{\infty}(-1)^{n}\left(\frac{4}{3 \pi}-\frac{\mathbf{H}_{2}(2 \pi n \lambda)}{\pi n \lambda}-\frac{8}{15 \pi}+\frac{\mathbf{H}_{3}(2 \pi n \lambda)}{(\pi n \lambda)^{2}}\right) .
\end{aligned}
$$

Next we have a closer look on the functions $\mathbf{H}_{2}$ and $\mathbf{H}_{3}$. We state some properties of these functions which will be needed in the following. Asymptotic expansions of $\mathbf{H}_{2}$ and $\mathbf{H}_{3}$ (see e.g. Abramowitz and Stegun [16] formula 12.1.29) yield

$$
\begin{aligned}
\left|\frac{4}{3 \pi}-\frac{\mathbf{H}_{2}(2 x)}{x}\right| \leqq \text { const } x^{-3 / 2}, \\
\left|\frac{8}{15 \pi}-\frac{\mathbf{H}_{3}(2 x)}{x^{2}}\right| \leqq \text { const } x^{-2} .
\end{aligned}
$$

Since $\frac{\mathbf{H}_{2}(x)}{x}$ and $\frac{\mathbf{H}_{3}(x)}{x^{2}}$ are entire functions, the bounds (4.2) hold for all $x \geqq 0$ and the second inequality implies

$$
\left|\frac{8}{15 \pi}-\frac{\mathbf{H}_{3}(2 x)}{x^{2}}\right| \leqq \text { const } x^{-3 / 2}
$$

for all $x \geqq 0$.

According to Abramowitz and Stegun (formula 12.1.9) the equalities

$$
\begin{gathered}
\frac{4}{3 \pi}-\frac{2 \mathbf{H}_{2}(x)}{x}=\frac{2 \mathbf{H}_{0}(x)}{x}-\frac{4 \mathbf{H}_{1}(x)}{x^{2}}, \\
\frac{8}{15 \pi}-\frac{4 \mathbf{H}_{3}(x)}{x^{2}}=\frac{4 \mathbf{H}_{1}(x)}{x^{2}}-\frac{16 \mathbf{H}_{2}(x)}{x^{3}},
\end{gathered}
$$


and the recurrence relation (formulae 12.1.9 and 12.1.10)

hold yielding

$$
\int_{0}^{x} \frac{\mathbf{H}_{n}(y)}{y^{n+1}} d y=\int_{0}^{x} \frac{\mathbf{H}_{n-1}(y)}{2 n y^{n}} d y-\frac{\mathbf{H}_{n}(x)}{2 n x^{n}}
$$

$$
\begin{aligned}
& \int_{0}^{x}\left(\frac{4}{3 \pi}-\frac{2 \mathbf{H}_{2}(y)}{y}\right) d y=\frac{2 \mathbf{H}_{1}(x)}{x}, \\
& \int_{0}^{x}\left(\frac{8}{15 \pi}-\frac{4 \mathbf{H}_{3}(y)}{y^{2}}\right) d y=\frac{4 \mathbf{H}_{2}(x)}{x^{2}} .
\end{aligned}
$$

Using (4.2) and (4.3) we get from (4.1)

$$
\begin{array}{r}
\sum_{l=0}^{\infty} \varrho_{l}^{H}(r) \leqq \frac{2 q}{3 \pi r} \lambda^{3}(r)+c_{d} \frac{1}{r} \lambda^{3 / 2}(r), \\
\sum_{l=0}^{\infty}\left(\frac{\alpha_{l}}{3}\left(\varrho_{l}^{H}\right)^{3}(r)+\frac{\left(l+\frac{1}{2}\right)^{2}}{r^{2}} \varrho_{l}^{H}(r)\right) \leqq \frac{2 q}{5 \pi r^{3}} \lambda^{5}(r)+c_{k} \frac{1}{r^{3}} \lambda^{7 / 2}(r),
\end{array}
$$

for some positive constants $c_{d}$ and $c_{k}$.

Now we are ready for

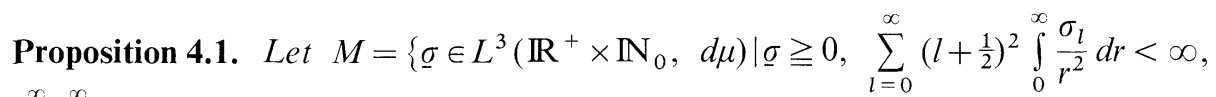
$\left.\sum_{l=0}^{\infty} \int_{0}^{\infty} \sigma_{l} d r<\infty\right\}$ (domain of the functional $\mathscr{E}_{Z} H$ ) where $d \mu$ is the Lebesgue measure in the first co-ordinate and the counting measure weighted by $\alpha_{l}$ in the second co-ordinate. For any positive constant $a^{\prime}$ holds $\underline{\varrho}^{H} \in M$ and $\sum_{l=0}^{\infty} \int_{0}^{\infty} \varrho_{l}^{H} d r \leqq Z-a^{\prime} Z^{1 / 2}$ for
sufficiently large $Z$.

Proof. The condition $\underline{\varrho}^{H} \in M$ requires finite kinetic energy and finite particle number. Since $\varrho_{l}^{H}(r)=0$, if $r \notin\left\{r \mid \lambda(r) \geqq \frac{1}{2}\right\}=\left[r_{1}, r_{2}\right]$, we get from (4.6):

$$
\begin{aligned}
& \int_{r_{1}}^{r_{2}} \frac{2 q}{5 \pi} \frac{\lambda^{5}(r)}{r^{3}} d r \leqq \int_{0}^{\infty} \frac{2 q L_{Z}^{5}(r)}{5 \pi r^{3}} d r=\mathrm{const} \int_{0}^{\infty}\left(\frac{\varrho_{\mathrm{TF}}(r)}{4 \pi r^{2}}\right)^{5 / 3} 4 \pi r^{2} d r<\infty \\
& \int_{r_{1}}^{1_{2}} \frac{\lambda^{7 / 2}}{r^{3}} d r \leqq \int_{1 /(4 Z)}^{\infty} \frac{(Z r)^{7 / 4}}{r^{3}} d r=\mathrm{const} Z^{2}<\infty \\
& \int_{r_{1}}^{r_{2}} \frac{2 q}{3 \pi r} \lambda^{3}(r) d r \leqq\left(1-a Z^{-1 / 2}\right) \int_{0}^{\infty} \varrho_{\mathrm{TF}}(r) d r=Z-a Z^{1 / 2}, \\
& \int_{r_{1}}^{r_{2}} c_{d} \frac{\lambda^{3 / 2}(r)}{r} d r \leqq c_{d} \int_{0}^{Z^{-1 / 3}} \frac{(Z r)^{3 / 4}}{r} d r+c_{d} \int_{Z^{-1 / 3}}^{\infty}\left(\frac{18 \pi}{q}\right)^{3 / 2} r^{-5 / 2} d r=b Z^{1 / 2}
\end{aligned}
$$

where the last equality defines the $Z$-independent constant $b$. Here we used (B.2), (B.4) and $1 / 4 Z \leqq r_{1}$, which follows from Lemma C.1. Combining the first two inequalities and the last two inequalities yields the proposed result, since $a$ may be chosen larger than $a^{\prime}+b$. 
We are now in a position to show that the density matrix has trace less than $Z$. We have

$$
\sum_{l=0}^{k^{\prime}-1}\left(\int_{0}^{x_{1}(l)} \varrho_{l} d r+\int_{x_{2}(l)}^{\infty} \varrho_{l} d r\right) \leqq b^{\prime} Z^{1 / 2}
$$

for some positive $b^{\prime}$ where we used Lemma C.1. Therefore we get

$$
\begin{aligned}
\operatorname{tr} d_{1} & =\sum_{l=0}^{L-1} q(2 l+1)(K-l)+\sum_{l=L}^{\infty} \int_{0}^{\infty} \varrho_{l} d r \\
& \leqq q K L^{2}+\sum_{l=0}^{\infty} \int_{0}^{\infty} \varrho_{l}^{H} d r+b^{\prime} Z^{1 / 2} \leqq Z+\left(q d+b^{\prime}-a^{\prime}\right) Z^{1 / 2} .
\end{aligned}
$$

Thus we obtain

Corollary 4.1. For some sufficiently large $a^{\prime}$ (and hence large a) and for sufficiently large $Z$

$$
\operatorname{tr} d_{1} \leqq Z
$$

Proof of Lemma 4.1. Because of the previous proposition we may insert our trial functions into the Hellmann functional. Using (4.1) we may write

$$
\begin{aligned}
\mathscr{E}_{Z}^{H}\left(\underline{\varrho}^{H}\right)= & \int_{0}^{\infty}\left(\frac{2 q \lambda^{5}}{5 \pi r^{3}}-\frac{Z}{r} \frac{2 q \lambda^{3}}{3 \pi r}\right) d r+\frac{1}{2} \int_{0}^{\infty} \int_{0}^{\infty}\left(\frac{2 q}{3 \pi}\right)^{2} \frac{\lambda^{3}(r) \lambda^{3}\left(r^{\prime}\right)}{r r^{\prime} \max \left\{r, r^{\prime}\right\}} d r^{\prime} d r \\
+ & \frac{1}{2} \int_{0}^{\infty} \int_{0}^{\infty} q^{2} \frac{\lambda^{3}(r) \lambda^{3}\left(r^{\prime}\right)}{r r^{\prime} \max \left\{r, r^{\prime}\right\}} \sum_{n=1}^{\infty}(-1)^{n}\left(\frac{4}{3 \pi}-\frac{\mathbf{H}_{2}(2 \pi n \lambda(r))}{\pi n \lambda(r)}\right) \\
& \cdot \sum_{m=1}^{\infty}(-1)^{m}\left(\frac{4}{3 \pi}-\frac{\mathbf{H}_{2}\left(2 \pi m \lambda\left(r^{\prime}\right)\right)}{\pi m \lambda\left(r^{\prime}\right)}\right) d r^{\prime} d r \\
+ & \int_{0}^{\infty} \sum_{n=1}^{\infty}(-1)^{n}\left(\frac{4}{3 \pi}-\frac{\mathbf{H}_{2}(2 \pi n \lambda(r))}{\pi n \lambda(r)}\right) \frac{q \lambda^{3}(r)}{r} \\
& \cdot\left(\frac{\lambda^{2}(r)}{r^{2}}-\frac{Z}{r}+\int_{0}^{\infty} \frac{2 q}{3 \pi} \frac{\lambda^{3}\left(r^{\prime}\right)}{r^{\prime} \max \left\{r, r^{\prime}\right\}} d r^{\prime}\right) d r \\
& -\int_{0}^{\infty} \sum_{n=1}^{\infty}(-1)^{n}\left(\frac{8}{15 \pi}-\frac{\mathbf{H}_{3}(2 \pi n \lambda)}{\left.(\pi n \lambda)^{2}\right)} \frac{q \lambda^{5}}{r^{3}} d r .\right.
\end{aligned}
$$

The first line yields $E_{Z}^{\mathrm{TF}}(Z)+O\left(Z^{4 / 3}\right)$, the second to the fifth line are terms of order $Z^{4 / 3}$ and the sixth line is a term of order $Z^{5 / 3}$. This will be shown in the following.

Using the definition of $\lambda$ and the scaling property of the Thomas-Fermi functional we find for the integrals in the first line of (4.8),

$$
\begin{gathered}
E_{Z}^{\mathrm{TF}}(Z)\left(-\left(1-a Z^{-1 / 2}\right)^{5 / 3}+\frac{7}{3}\left(1-a Z^{-1 / 2}\right)-\frac{1}{3}\left(1-a Z^{-1 / 2}\right)^{2}\right) \\
=E_{Z}^{\mathrm{TF}}(Z)(1+O(1 / Z)) .
\end{gathered}
$$

The double integral in the second and third line of (4.8) may be enlarged using the absolute value of the summands, the first of the inequalities (4.2) and 
$\frac{1}{\max \left\{r, r^{\prime}\right\}} \leqq \frac{1}{\sqrt{r r^{\prime}}}$. This yields the upper bound

$$
\frac{1}{2}\left(\sum_{n=1}^{\infty} \int_{0}^{\infty} \frac{q \lambda^{3 / 2}(r)}{(2 \pi n r)^{3 / 2}} d r\right)^{2} \leqq \text { const } Z^{4 / 3},
$$

which follows from inserting $\lambda$ and using the scaling property of $\varrho_{\mathrm{TF}}$.

Using the Thomas-Fermi equation we find

$$
\begin{aligned}
\frac{\lambda^{2}}{r^{2}}-\frac{Z}{r}+\int_{0}^{\infty} \frac{2 q}{3 \pi} \frac{\lambda^{3}\left(r^{\prime}\right)}{r^{\prime} \max \left\{r, r^{\prime}\right\}} d r^{\prime}= & \frac{\lambda^{2}}{r^{2}}\left(1-\left(1-a Z^{-1 / 2}\right)^{-2 / 3}\right) \\
& -a Z^{-1 / 2} \int_{0}^{\infty} \frac{\varrho_{\mathrm{TF}}}{\max \left\{r, r^{\prime}\right\}} d r^{\prime}
\end{aligned}
$$

Hence we have the following upper bound for the fourth and fifth line of (4.8),

$$
\begin{gathered}
\sum_{n=1}^{\infty} \int_{0}^{\infty} \frac{\text { const } q \lambda^{3 / 2}(r)}{n^{3 / 2} r} a Z^{-1 / 2} \int_{0}^{\infty} \frac{\varrho_{\mathrm{TF}}}{\max \left\{r, r^{\prime}\right\}} d r^{\prime} d r \\
+\sum_{n=1}^{\infty}(-1)^{n}\left(1-\left(1-a Z^{-1 / 2}\right)^{-2 / 3}\right) \int_{0}^{\infty}\left(\frac{4}{3 \pi}-\frac{\mathbf{H}_{2}(2 \pi n \lambda)}{\pi n \lambda}\right) \frac{q \lambda^{5}}{r^{3}} d r .
\end{gathered}
$$

The first term in this sum is of order $Z^{4 / 3}$. The second term is similar to the one which is left in (4.8). They are evaluated after splitting the range of integration at $R_{1} Z^{-1 / 3}$ for some positive sufficiently small $R_{1}$. Upper bounds for the integrals over $\left(R_{1} Z^{-1 / 3}, \infty\right)$ are obtained by use of scaling and the bounds (4.2) yielding terms of order $Z^{4 / 3}$ and $Z^{5 / 3}$, respectively. In order to evaluate the integrals over $\left(0, R_{1} Z^{-1 / 3}\right)$ we carry out the transformation $2 \pi n \lambda(r)=y$. Moreover, according to Appendix B,

$$
\left|\frac{\lambda^{5}}{r^{3} \lambda^{\prime}}-2 Z^{2}\left(1-a Z^{-1 / 2}\right)^{4 / 3}\right| \leqq \operatorname{const} Z^{7 / 3} r \leqq \operatorname{const} Z^{4 / 3} \frac{y^{2}}{n^{2}}
$$

Hence

$$
\begin{aligned}
& \left|\sum_{n=1}^{\infty}(-1)^{n}\left(1-\left(1-a Z^{-1 / 2}\right)^{-2 / 3}\right) \int_{0}^{R_{1} Z^{-1 / 3}}\left(\frac{4}{3 \pi}-\frac{\mathbf{H}_{2}(2 \pi n \lambda)}{\pi n \lambda}\right) \frac{q \lambda^{5}}{r^{3}} d r\right| \\
& \quad \leqq \text { const } Z^{-1 / 2} \sum_{n=1}^{\infty}\left(\frac{q Z^{2}}{\pi n}\left|\int_{0}^{B}\left(\frac{4}{3 \pi}-\frac{2 \mathbf{H}_{2}(y)}{y}\right) d y\right|+\frac{\operatorname{const} Z^{4 / 3}}{n^{3}} \int_{0}^{B} y^{1 / 2} d y\right) \\
& \quad \leqq \sum_{n=1}^{\infty} \operatorname{const}\left(Z^{3 / 2} \frac{2 \mathbf{H}_{1}(B)}{n B}+\frac{Z^{5 / 6} B^{3 / 2}}{n^{3}}\right)=O\left(Z^{4 / 3}\right),
\end{aligned}
$$

and

$$
\begin{aligned}
& \left|\sum_{n=1}^{\infty}(-1)^{n} \int_{0}^{R_{1} Z^{-1 / 3}}\left(\frac{8}{15 \pi}-\frac{\mathbf{H}_{3}(2 \pi n \lambda)}{(\pi n \lambda)^{2}}\right) \frac{q \lambda^{5}}{r^{3}} d r\right| \\
& \quad \leqq \sum_{n=1}^{\infty}\left(\frac{q Z^{2}}{\pi n}\left|\int_{0}^{B}\left(\frac{8}{15 \pi}-\frac{4 \mathbf{H}_{3}(y)}{y^{2}}\right) d y\right|+\mathrm{const} \frac{Z^{4 / 3}}{n^{3}} \int_{0}^{B} d y\right) \\
& \quad \leqq \sum_{n=1}^{\infty} \operatorname{const}\left(Z^{2} \frac{4 \mathbf{H}_{2}(B)}{n B^{2}}+\frac{Z^{4 / 3} B}{n^{3}}\right)=O\left(Z^{5 / 3}\right),
\end{aligned}
$$


where $B=2 \pi n \lambda\left(R_{1} Z^{-1 / 3}\right)$. Here we used (4.5), $B=O\left(n Z^{1 / 3}\right)$ and the facts that $\mathbf{H}_{1}(x)$ as well as $\frac{\mathbf{H}_{2}(x)}{x}$ are bounded. This is the result which was proposed in Lemma 4.1.

We may now collect all the results obtained in this paper. According to our general strategy they just constitute the proof of Theorem 1.1. We conclude with the remark that our result also shows that the Hellmann-Weizsäcker functional with the correction term in front of the $\varrho_{l}^{3}$-term and with the first $L$ angular momentum densities substituted by densities of Bohr orbitals (right hand side of Eq. (2.3)) is a strict upper bound (no error terms) on the ground state energy of atoms, and has an infimum with the correct behavior as a function of the particle number in first and second order.

We conclude with the note that the analogues of (4.6) that would be required for a lower bound follow from a simple convexity argument instead of the Poisson summation used here.

\section{Appendix A}

Let $\varphi$ be the solution of the Thomas-Fermi equation $\varphi^{\prime \prime}=\frac{\varphi^{3 / 2}}{x^{1 / 2}}$ on $\mathbb{R}^{+}$with the boundary conditions $\varphi(0)=1$ and $\lim _{x \rightarrow \infty} \varphi(x)=0$. Now define the function $\hat{L}(x)=\sqrt{x \varphi(x)}$. We shall show the following properties of $\hat{L}$ :

\section{Lemma A.1.}

1. $\hat{L}^{\prime \prime}$ has exactly one zero $x_{w}$. This is a point of inflexion of $\hat{L}$, where the curvature turns from concave to convex.

2. $\hat{L}^{\prime}$ has exactly one zero $x_{m}$. This is a maximum of $\hat{L}$.

Proof. First we remark that $x^{3} \varphi(x)$ is strictly monotone increasing. This follows from Theorems 2.8 and 2.10 of Lieb [4], where Lieb's $\phi$ is related to $\varphi$ by $\phi(r)=\frac{Z}{r} \varphi(b r)$ with $b=Z^{1 / 3}\left(\frac{2 q}{3 \pi}\right)^{2 / 3}$. Theorem 2.10 asserts the monotonicity of $x^{3} \varphi(x)$, while the real analyticity of $\phi$ and hence of $x^{3} \varphi(x)$, stated in Theorem 2.8, assures the strictness of the monotonicity. Moreover Theorem 2.10 implies that $\lim _{x \rightarrow \infty} x^{3} \varphi(x)=144$.

Therefore we have that $\hat{L}(0)=0, \lim _{x \rightarrow \infty} \hat{L}(x)=0$ and that $\hat{L}(x)>0$ for $0<x<\infty$. Hence $\hat{L}$ has at least one point of inflexion, where the curvature turns from concave to convex, and one maximum. Now we consider the possible zeros of $\hat{L}^{\prime \prime}$. If $\hat{L}^{\prime \prime}\left(x_{w}\right)=0$, then

$$
2 x_{w}^{2} \varphi\left(x_{w}\right) \varphi^{\prime \prime}\left(x_{w}\right)=\left(x_{w} \varphi^{\prime}\left(x_{w}\right)-\varphi\left(x_{w}\right)\right)^{2} .
$$

This yields

$$
\begin{aligned}
\hat{L}^{\prime \prime \prime}\left(x_{w}\right) & =\frac{1}{2 \sqrt{x_{w} \varphi\left(x_{w}\right)}}\left(3 \varphi^{\prime \prime}\left(x_{w}\right)+x_{w} \varphi^{\prime \prime \prime}\left(x_{w}\right)\right) \\
& =\frac{3 \sqrt{2} \varphi\left(x_{w}\right)}{4 x_{w}}\left(\frac{8}{3 \sqrt{2}}-x_{w}^{3 / 4} \varphi^{1 / 4}\left(x_{w}\right)\right),
\end{aligned}
$$


using the differential equation. By the above there is exactly one point $x_{0}$ with $x_{0}^{3} \varphi\left(x_{0}\right)=\left(\frac{8}{3 \sqrt{2}}\right)^{4}$. At points $x_{w}$ with $x_{w}<x_{0}$ the curvature of $\hat{L}$ turns therefore from concave to convex and at points $x_{w}$ with $x_{w}>x_{0}$ the curvature of $\hat{L}$ turns from convex to concave. Hence there can be at most three points with $\hat{L}^{\prime \prime}\left(x_{w}\right)=0$. One to the left, one to the right of $x_{0}$ and one at $x_{0}$. We now exclude the second and third possibility. The existence of an inflexion point $x_{w}$ with $x_{w}>x_{0}$ is impossible, since $\hat{L}$ would then be concave for large $x$. If $\hat{L}^{\prime \prime}\left(x_{0}\right)=0$, i.e. $x_{w}=x_{0}$, we find $\hat{L}^{\prime \prime \prime}\left(x_{0}\right)=0$ and $\hat{L}^{(i v)}\left(x_{0}\right)<0$, and therefore $x_{0}$ would be a maximum of $\hat{L}^{\prime \prime}$. Hence $\hat{L}$ would be concave for large $x$ in this case, too. Therefore $\hat{L}^{\prime \prime}$ has exactly one zero. This is an inflexion point of $\hat{L}$, where the curvature turns from concave to convex.

Analogously we have for the points $x_{m}$ with $\hat{L}^{\prime}\left(x_{m}\right)=0$ the condition $x_{m} \varphi^{\prime}\left(x_{m}\right)=-\varphi\left(x_{m}\right)$ yielding

$$
\hat{L}^{\prime \prime}\left(x_{m}\right)=\frac{\varphi^{1 / 2}\left(x_{m}\right)}{2 x_{m}^{3 / 2}}\left(x_{m}^{3 / 2} \varphi^{1 / 2}\left(x_{m}\right)-2\right) .
$$

Again there is exactly one point $x_{1}$ with $x_{1}^{3} \varphi\left(x_{1}\right)=4$. If $x_{m}<x_{1}$, then there is a maximum at $x_{m}$. If $x_{m}>x_{1}$, then there is a minimum at $x_{m}$. Again there can be at most three points with $\hat{L}^{\prime}\left(x_{m}\right)=0$. Similar arguments as above exclude the cases $x_{m} \geqq x_{1}$. Hence $\hat{L}^{\prime}$ has exactly one zero yielding a maximum of $\hat{L}$.

Now we are interested in upper and lower bounds on $\hat{L}$. Since $\varphi(x) \leqq 1$ and $\varphi(x) \leqq \frac{144}{x^{3}}$ we have immediately

$$
\hat{L}(x) \leqq \sqrt{x} \text { and } \hat{L}(x) \leqq \frac{12}{x} .
$$

For $x \leqq x_{m}$ we have $\varphi(x) \geqq \varphi\left(x_{m}\right)$, since $\varphi$ is strictly monotone decreasing. Hence

$$
\hat{L}(x) \geqq \sqrt{x \varphi\left(x_{m}\right)} \text { for } 0 \leqq x \leqq x_{m} .
$$

This lower bound may be strengthened in the following way:

$$
\hat{L}(x) \geqq \sqrt{x}\left(1+\varphi^{\prime}(0) x\right),
$$

using Taylor's theorem. By Lieb's Theorem 2.10 case (iii) we obtain

$$
\hat{L}(x) \geqq \frac{\sqrt{x_{m}^{3} \varphi\left(x_{m}\right)}}{x} \text { for } \quad x_{m} \leqq x<\infty .
$$

Moreover we give bounds for $\hat{L}^{\prime}$.

$$
\begin{aligned}
& \hat{L}^{\prime}(x) \leqq \frac{1}{2 \sqrt{x}}\left(1-2 x \varphi^{\prime}(0)\right) \text { for } \quad-x \varphi^{\prime}(0) \leqq \frac{1}{2}, \\
& \hat{L}^{\prime}(x) \geqq \frac{1}{2 \sqrt{x}}\left(1+2 x \varphi^{\prime}(0)\right) .
\end{aligned}
$$

In a similar way as in the proof of de l'Hospital's rule, we may conclude from $\lim _{x \rightarrow \infty} x \hat{L}(x)=12$ that $\lim _{x \rightarrow \infty} x^{2} \hat{L}^{\prime}(x)=-12$ holds. Hence a positive constant $A$ exists with $\hat{L}^{\prime}(x) \leqq-\frac{A}{x^{2}}$ for $x \geqq x_{w}$. 


\section{Appendix B}

We define a function $L_{Z}$ by

$$
L_{Z}(r)=Z^{1 / 3}\left(\frac{3 \pi}{2 q}\right)^{1 / 3} \hat{L}(b r) \quad \text { with } \quad b=Z^{1 / 3}\left(\frac{2 q}{3 \pi}\right)^{2 / 3} .
$$

One easily finds the following relation of $L_{Z}$ to the radial Thomas-Fermi density $\varrho_{\mathrm{TF}}$ :

$$
L_{Z}(r)=\left(\frac{3 \pi}{2 q} r \varrho_{\mathrm{TF}}(r)\right)^{1 / 3} .
$$

Therefore we may write the Thomas-Fermi energy in the following way:

$$
\begin{aligned}
E_{Z}^{\mathrm{TF}}(Z)= & \frac{2 q}{5 \pi} \int_{0}^{\infty} \frac{L_{Z}^{5}(r)}{r^{3}} d r-\frac{2 q}{3 \pi} \int_{0}^{\infty} \frac{Z}{r} \frac{L_{Z}^{3}(r)}{r} d r \\
& +\frac{1}{2} \int_{0}^{\infty} \int_{0}^{\infty}\left(\frac{2 q}{3 \pi}\right)^{2} \frac{L_{Z}^{3}(r) L_{Z}^{3}\left(r^{\prime}\right)}{r r^{\prime} \max \left\{r, r^{\prime}\right\}} d r^{\prime} d r .
\end{aligned}
$$

Furthermore we have immediately the following scaling $L_{Z}(r)=Z^{1 / 3} L_{1}\left(Z^{1 / 3} r\right)$ yielding the existence of a single maximum of $L_{Z}$ at $R Z^{-1 / 3}$ and of a single zero of $L_{Z}^{\prime \prime}$ at $W Z^{-1 / 3}$ for some $R$ and $W$ in $\mathbb{R}^{+}$. Finally we collect some further properties of $L_{Z}$ :

$$
\begin{aligned}
& L_{Z}^{(n)}(r)=Z^{(n+1) / 3}\left(\frac{2 q}{3 \pi}\right)^{(2 n-1) / 3} \hat{L}^{(n)}(b r), \\
& L_{Z}(r) \leqq \sqrt{Z r}, \\
& L_{Z}(r) \leqq \frac{18 \pi}{q r}, \\
& L_{Z}(r) \geqq \sqrt{\varphi\left(x_{m}\right) Z r} \text { for } \quad b r \leqq x_{m}, \\
& L_{Z}(r) \geqq \sqrt{Z r}\left(1+\varphi^{\prime}(0)\left(\frac{2 q}{3 \pi}\right)^{2 / 3} Z^{1 / 3} r\right), \\
& L_{Z}(r) \geqq \frac{3 \pi}{2 q r} \sqrt{x_{m}^{3} \varphi\left(x_{m}\right)} \quad \text { for } \quad b r \geqq x_{m}, \\
& L_{Z}^{\prime}(r)=\frac{1}{2} \sqrt{\frac{Z}{r}}\left(1+O\left(Z^{1 / 3} r\right)\right) \quad \text { for } \quad b r \leqq \frac{-1}{2 \varphi^{\prime}(0)} .
\end{aligned}
$$

The point $x_{m}$ is defined in Appendix A.

\section{Appendix C}

In this appendix we deal with important properties of $r_{1}(l), r_{2}(l), x_{1}(l)$ and $x_{2}(l)$, the points that we introduced in the definition of the trial density for the HellmannWeizsäcker functional. 
Lemma C.1. For sufficiently large $Z$ the following holds:
i) $\frac{\left(l+\frac{1}{2}\right)^{2}}{Z} \leqq r_{1}(l) \leqq \frac{\left(l+\frac{1}{2}\right)^{2}}{Z} \frac{4}{\varphi\left(x_{m}\right)}$,
ii) $\frac{3 \pi}{2 q(2 l+1)} \sqrt{x_{m}^{3} \varphi\left(x_{m}\right)} \leqq r_{2}(l) \leqq \frac{36 \pi}{q(2 l+1)}$,
iii) $x_{1}(l) \leqq R Z^{-1 / 3}-$ const $Z^{-1 / 2} \leqq R Z^{-1 / 3}+$ const $Z^{1 / 2} \leqq x_{2}(l)$,
iv) $R_{l}\left(x_{1}(l)\right) \leqq T\left(l+\frac{1}{2}\right)$
v) const $\frac{\left(l+\frac{1}{2}\right)}{Z^{2 / 3}} \min \left\{\left(l+\frac{1}{2}\right)^{2}, Z^{1 / 2}\right\} \leqq R_{l}\left(x_{2}(l)\right) \leqq$ const $Z^{1 / 3}$,

where $0 \leqq l \leqq k^{\prime}$ in i) and ii) while $0 \leqq l \leqq k^{\prime}-1$ in iii), iv) and v). For abbreviation we wrote $R_{l}(r)=\left[\lambda^{2}(r)-\left(l+\frac{1}{2}\right)^{2}\right]_{+}$.

Proof. The inequalities i) and ii) follow immediately from (B.4) and from $\left(1-a Z^{-1 / 2}\right)^{1 / 3} \geqq \frac{1}{2}$ for sufficiently large $Z$.

For iii) it is sufficient to consider the case $l=k^{\prime}-1$, since $x_{1}(l)<x_{1}(l+1)$ and $x_{2}(l)>x_{2}(l+1)$. Using the mean value theorem we find

$$
\left(r_{1}\left(k^{\prime}-1\right)-R Z^{-1 / 3}\right)^{2}=\frac{2\left(k-k^{\prime}+1\right)}{-\lambda^{\prime \prime}\left(r^{\prime}\right)},
$$

with $r^{\prime} \in\left[r_{1}\left(k^{\prime}-1\right), R Z^{-1 / 3}\right]$. Since $k-k^{\prime}+1>1$ and $-\lambda^{\prime \prime}\left(r^{\prime}\right) \leqq$ const $Z$, we have $r_{1}\left(k^{\prime}-1\right) \leqq R Z^{-1 / 3}-$ const $Z^{-1 / 2}$. The first part of iii) follows then immediately for large $Z$. The second part of iii) follows analogously.

To show iv) we remark firstly that $\left(\hat{L}^{2}\right)^{\prime} \leqq 1$, and hence $\left(L_{Z}^{2}\right)^{\prime} \leqq Z$ holds. Again the mean value theorem yields $\lambda^{2}\left(x_{1}(l)\right) \leqq\left(l+\frac{1}{2}\right)^{2}+T \frac{\left(l+\frac{1}{2}\right)}{Z} Z$, which is the desired result.

The second inequality in v) is proven analogously using $\left(L_{Z}^{2}\right)^{\prime} \geqq-$ const $Z$. For the first inequality of $\mathrm{v})$ we find $\lambda\left(x_{2}(l)\right) \geqq\left(l+\frac{1}{2}\right)+\left(x_{2}(l)-r_{2}(l)\right) \max \left\{\lambda^{\prime}\left(x_{2}(l)\right)\right.$, $\left.\lambda^{\prime}\left(r_{2}(l)\right)\right\}$. It suffices to consider the cases $x_{2}(l) \leqq W Z^{-1 / 3}$ and $r_{2}(l) \geqq W Z^{-1 / 3}$. Since we can find a positive constant $A$ to bound $\hat{L}^{\prime}(x)$ from above by $\frac{-A}{x^{2}}$ for $x>x_{w}$ (see Appendix A), we have $\lambda^{\prime}\left(r_{2}(l)\right) \leqq-$ const $\left(l+\frac{1}{2}\right)^{2}$ for sufficiently large $Z$. Furthermore we find $\lambda^{\prime}\left(x_{2}(l)\right) \leqq$ const $Z^{-1 / 2} \lambda^{\prime \prime}\left(r^{\prime}\right)$ with $r^{\prime} \in\left[R Z^{-1 / 3}\right.$, $R Z^{-1 / 3}+$ const $\left.Z^{-1 / 2}\right]$. For sufficiently large $Z$ we have $\lambda^{\prime \prime}\left(r^{\prime}\right) \leqq-$ const $Z$. Hence $\lambda^{\prime}\left(x_{2}(l)\right) \leqq-$ const $Z^{1 / 2}$ yielding the proposed result.

Acknowledgements. We are grateful to Prof. A.M.K. Müller and Prof. E. Richter for their support of this work. One of us (H.S.) thanks Prof. E.H. Lieb for some critical remarks and suggestions.

\section{References}

1. Hughes, W.: An atomic energy lower bound that proves Scott's correction. Dissertation, Department of Mathematics, Princeton University 1986

2. Scott, J. M.C.: The binding energy of the Thomas-Fermi atom. Phil. Mag. 43, 859-867 (1952) 
3. Lieb, E.H., Simon, B.: The Thomas-Fermi theory of atoms, molecules and solids. Adv. Math. 23, 22-116 (1977)

4. Lieb, E.H.: Thomas-Fermi and related theories of atoms and molecules. Rev. Mod. Phys. 53, 603-641 (1981)

5. Thirring, W.: A lower bound with the best possible constant for Coulomb hamiltonians. Commun. Math. Phys. 79, 1-7 (1981)

6. Schwinger, J.: Thomas-Fermi model: The leading correction. Phys. Rev. A22, 1827-1832 (1980)

7. Englert, B.-G., Schwinger, J.: Statistical atom: Handling the strongly bound electrons. Phys. Rev. A29, 2331-2338 (1984)

8. Bander, M.: Corrections to the Thomas-Fermi model of the atom. Ann. Phys. (New York) 144, 1-14 (1982)

9. Siedentop, H.K.H., Weikard, R.: On some basic properties of density functionals for angular momentum channels, accepted for publication in Rep. Math. Phys.

10. Siedentop, H.K.H., Weikard, R.: On the behavior of the infimum of the HellmannWeizsäcker functional, Technical Report (1986)

11. Siedentop, H.K.H., Weikard, R.: On the leading energy correction for the statistical model of the atom: Non-interacting case. Abh. Braunschw. Wiss. Ges. (Germany) 38, 145-158 (1986)

12. Lieb, E.H.: Variational principle for many-fermion systems. Phys. Rev. Lett. 46, 457-459 (1981); Erratum. Phys. Rev. Lett. 47, 69 (1981)

13. Erdélyi, A., Magnus, W., Oberhettinger, F., Tricomi, F. G.: Higher transcendal functions. I. New York: McGraw Hill 1953

14. Englert, B.-G., Schwinger, J.: Atomic-binding-energy oscillations. Phys. Rev. A32, 47-63 (1985)

15. Bochner, S.: Vorlesungen über Fouriersche Integrale. New York: Chelsea 1948

16. Abramowitz, M., Stegun, I.: Handbook of mathematical functions. New York: Dover 1965

Communicated by B. Simon

Received December 3, 1986; in revised form April 23, 1987 